\title{
Progression of acute hydrocephalus in subarachnoid haemorrhage: a case report documented by serial CT scanning
}

\author{
Gabriel J E Rinkel, Eelco F M Wijdicks, Lino M P Ramos, Jan van Gijn
}

\begin{abstract}
A patient with a perimesencephalic subarachnoid haemorrhage gradually developed, within the first day, progressively impaired consciousness, small non-reactive pupils, and / defective upward gaze. Three successive CT scans showed progressive enlargement of the lateral and third ventricles. Ventricular shunting resulted in complete recovery.
\end{abstract}

Impairment of consciousness in the first few hours after aneurysmal subarachnoid haemorrhage (SAH) can be a direct effect of the haemorrhage itself or of hydrocephalus. Recently the distinctive clinical features of acute hydrocephalus have been outlined: a gradual decline of the level of consciousness and small nonreactive pupils, with or without impaired upward gaze, and with otherwise intact brainstem reflexes. ${ }^{1}$ These findings have been confirmed in a separate study. ${ }^{2}$ Until now, all correlations were based on single admission CT scans. In this case report we document the gradual development of the clinical as well as the radiological signs of hydrocephalus.

\section{Case report}

A 57 year old previously healthy man had a sudden and severe headache, radiating to the neck, while he was refereeing a soccer game. $\mathrm{He}$ did not lose consciousness. He was transferred to our service after a lumbar puncture performed at another hospital revealed grossly bloody spinal fluid.

On examination there was no alteration in the level of consciousness. Neck stiffness was present, otherwise the neurological examination was normal. A CT scan taken four hours after the ictus showed blood in the basal cisterns, predominantly around the mesencephalon, ${ }^{3}$ and ventricular dilatation (figure 1 , upper row), as measured by the bicaudate index (the width of the frontal horns at the level of the caudate nuclei, divided by the width of the brain at the same level). ${ }^{1}$ The relative size (the actual bicaudate index divided by the upper limit for age of the bicaudate index) was $1 \cdot 37$. The relative third ventricular $\operatorname{size}^{4}$ was 1.66 .

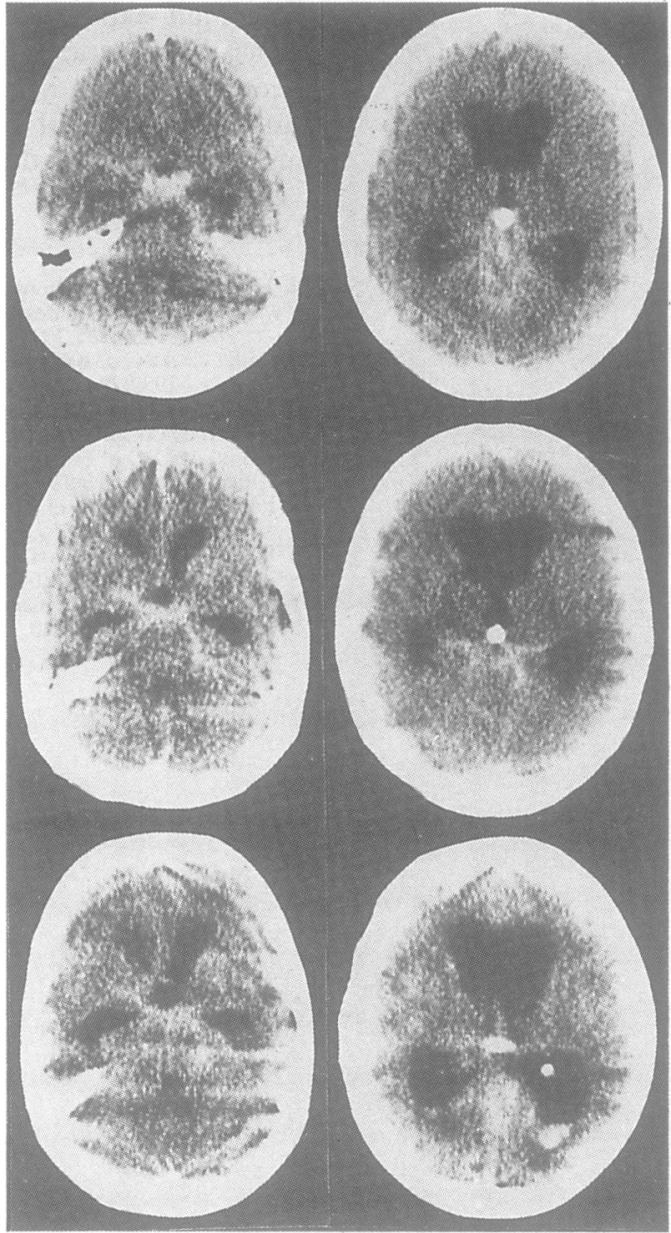

Figure 1 Non-contrast CT scans. Left: basal cistern level. Right: pineal level. Upper row: four hours after the ictus. Haemorrhage in the basal cisterns, with most of the blood located in the interpeduncular fossa and the left ambient cistern (relative size of lateral ventricles 1.37). Middle row: nine hours after the ictus. Blood in the basal cisterns unchanged (relative size of lateral ventricles 1.42). Lower row: 28 hours after the ictus. Increased hydrocephalus with ventricular sedimentation (relative size of lateral ventricles 1.84).

He gradually became disoriented and less responsive. He opened his eyes only when spoken to, obeyed commands, but spoke incoherently. A repeated CT scan, taken nine hours after the bleeding, showed only slight progression of the degree of hydrocephalus, with a relative ventricular size of 1.42 (fig 1 , middle row). The next day the deterioration continued (fig 2). He opened his eyes only to noxious stimuli, did not obey commands but 
Figure 2 Time course of relative size of lateral ventricles and third ventricle, in relation with ventricular drainage and Glasgow Coma Sum Scores.

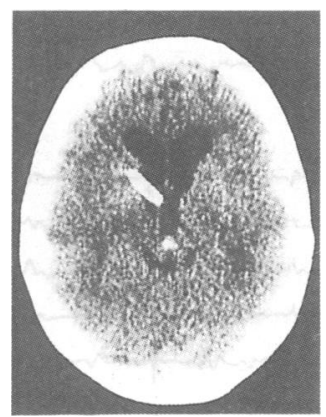

Figure 3 Five weeks after the ictus. Shunt in right lateral ventricle. Reduction of hydrocephalus (relative size of lateral ventricles $1 \cdot 20)$.

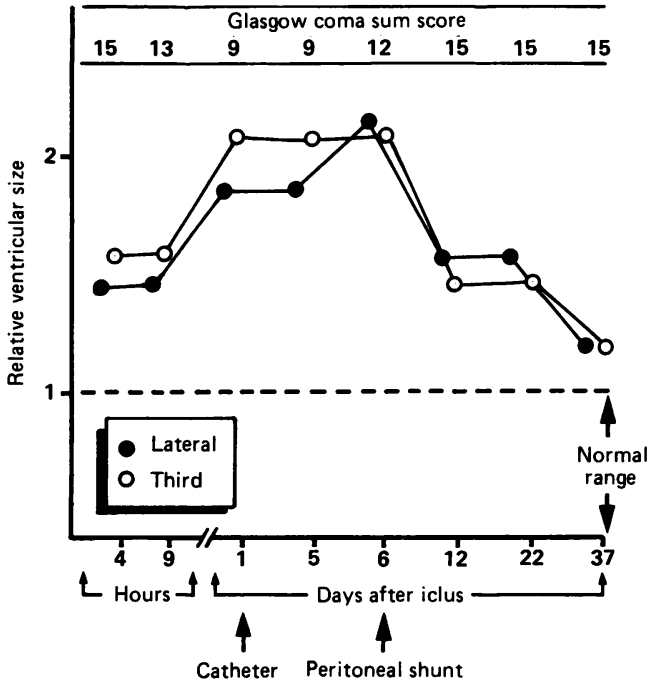

only localised to pain, and produced only sounds. Neck stiffness was more marked, the pupils were small and non-reactive, and reflex upward gaze was impaired. Muscle tone and tendon jerks were increased, with bilateral extensor plantar responses. No metabolic disturbances were found that could account for this deterioration. A third CT scan, 28 hours after the bleeding, showed considerable enlargement of the ventricles, with a relative size of 1.84 (fig 1, lower row). A catheter for monitoring pressure was inserted; the ventricular pressure at operation was $50 \mathrm{~cm}$ water.

During the following five days his clinical situation remained unaltered. To keep the ventricular pressure at about $20 \mathrm{~cm}$ water some cerebrospinal fluid had to be withdrawn repeatedly, as elevations to $40 \mathrm{~cm}$ water were noted.

A ventriculoperitoneal shunt was inserted six days after the bleeding. Postoperatively, the level of consciousness improved within hours and returned to normal in days. The ocular signs disappeared, together with increased muscle tone and reflex abnormalities. A control CT scan on day 37 showed a reduction of the ventricular size to almost normal (fig 3; relative ventricular size 1.20). Apart from a pulmonary infection, which was successfully treated, no further complications occurred. Four-vessel angiography was performed and showed only a small aneurysm of the pericallosal artery; this was distant from the subarachnoid clot and was not thought to be the origin of the bleed, although this could not be completely excluded. The patient has returned to a normal life.

\section{Discussion}

To our knowledge, this is the first reported case in which the development of hydrocephalus after SAH has been documented by serial CT scanning. The admission CT scan already showed marked enlargement of the lateral and third ventricles, although at that time the level of consciousness was normal, and no ocular signs were yet present. With progression of the ventricular dilatation, the level of conscious- ness became impaired first, later followed by nonreactive pupils and defective upward gaze. Complete recovery occurred after cerebrospinal fluid (CSF) diversion, when the ventricular size had returned to almost normal.

The importance of recognising acute hydrocephalus after SAH is now well established. Our patient illustrates that the initial phase of enlargement of the ventricles can be clinically silent. Impairment of consciousness occurs after further enlargement of the ventricles, provided that the patient is still alert after the initial haemorrhage. Probably at some stage the enlarging ventricles interfere with the cerebral perfusion pressure, with a depressed level of consciousness as a result. ${ }^{12}$ Only a small proportion of patients with acute hydrocephalus exhibit a Parinaud syndrome (nonreactive pupils and defective upward gaze with otherwise intact brainstem reflexes). ${ }^{1}$ Apparently, expansion of the rostral part of the aquaduct occurs at an even later stage, leading to dysfunction of the periaquaductal grey matter. Another important observation was that the enlargement of the third ventricle paralleled that of the lateral ventricles. This is in contrast with the rostro-caudal sequence of ventricular enlargement suggested by Milhorat. ${ }^{2}$ On the other hand, the CSF flow was probably obstructed at the level of the tentorial hiatus in this patient, whereas in aneurysmal haemorrhage the occurrence of hydrocephalus is closely associated with intraventricular bleeding. ${ }^{1}$

Immediate and significant improvement after ventricular drainage has also been reported by van $\mathrm{Gijn}^{1}$ in all nine shunted patients, and by Milhorat in 25 out of 35 shunted patients $(71 \%)$. But the improved outcome in our patient is unusual, as in the van Gijn study ${ }^{1}$ eight out of nine shunted patients died, four from rebleeding and four from cerebral infarction, and in the study by Milhorat $17 \%$ of the patients died. The most probable explanation is that this patient suffered a perimesencephalic haemorrhage, which is a non-aneurysmal and perhaps nonarterial type of haemorrhage around the midbrain. ${ }^{3}$ This type of SAH has an invariably good outcome, without rebleeding or cerebral infarction. Although a small pericallosal aneurysm was found, we believe it was coincidental, in view of the distribution of blood on CT scan. We thank T Dokkum, $H \mathrm{~J}$ van Zuylen and $\mathrm{P} W$ Hanlo, who
helped in the care of this patient, and Mrs E Budelman-

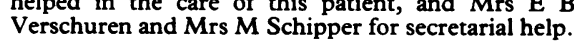

1 van Gijn J, Hijdra A, Wijdicks EFM, Vermeulen $M$ van Crevel $\mathrm{H}$. Acute hydrocephalus after aneurysma subarachnoid haemorrhage. J Neurosurg 1985;63:355-62.

2 Milhorat TH. Acute hydrocephalus after aneurysma subar subarachnoid haemorrhage. Neurosurgery 1987;20:15-20. van Gijn J, van Dongen KJ, Vermeulen M, Hijdra A Perimesencephalic hemorrhage: a nonaneurysmal and benign form of subarachnoid haemorrhage. Neurology

4 Wijdicks EFM, van Dongen KJ, van Gijn J, Hijdra A Vermeulen $M$. Enlargement of the third ventricle and hyponatraemia in aneurysmal subarachnoid haemorrhage. J Neurol Neurosurg Psychiatry 1988;51:516-20. 